\title{
Studien über die Menge des Glomerulusfiltrats.
}

\section{Glomerulusfiltrat bei Beriberi.}

Von

Fusakichi Nakazawa und Hyoe Kusakari.

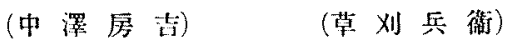

(Aus der Medizinischen Klinik von Prof. Dr. T. Kato

an der Toholu Reichsuniversität zu Sendai.)

Die Teilfunktionen der einzelnen Abschnitte der Niere kennen zu lernen, bereitet erhebliche Schwierigkeiten, da der Mechanismus der Harnbildung noch sehr wenig geklürt ist.

Nach der Veröffentlichung von P. B. Rehberg, ${ }^{1)}$ welcher vor allem die verschiedenen Probleme der Nierenfunktionen aufzuhellen gesucht hat, ist es möglich, die Menge des sogenannten Glomerulusfiltrats nach einer einfachen Methode zu bestimmen, indem man sie durch Berechnung der Konzentration des Kreatinins in dem innerhalb eines bestimmten Zeitraums nach oraler Verabreichung von Kreatinin aufgefangenen Harn und im nach derselben Zeit entnommenen Blutplasma und der Menge des betreffenden Harns findet. Hier werden wir kurz die zwei grundlegenden Theorien und das Prinzip der Messung beschreiben.

1. Dass die Glomeruli dazu dienen, Flüssigkeit aus dem Blut herauszufiltrieren, wird wohl jetzt von den meisten Physiologen anerkannt und nach der Ansicht von Cushny, ${ }^{2}$ Starling, ${ }^{3)}$ Richards ${ }^{4)}$ u. a. ist es sehr wahrscheinlich, dass das Glomerulusfiltrat nichts anderes als das enteiweisste Blutplasma ist, oder mindestens dem Ultrafiltrat des Bluts sehr ähnlich sein muss. Wenn man also die Konzentration des Kreatinins im Blutplasma (KrP) misst, dann kann man diese als dessen Konzentration im Glomerulusfiltrat ( $\mathrm{KrG})$ ansehen.

$$
\mathrm{KrP} \fallingdotseq \mathrm{KrG}
$$

1) Rehberg, Bioch. Journ., 1926, 20, 487 und 461; Ztrlbl. f. inn. Med., 1929, 50, 367.

2) Cushny, The Secretion of the Urine, 2. Autl, London 1926, S. 52.

3) St arling und Verney, Proc. Roy. Soc. Lond. B., 1925, 97, 321.

4) Richards und Schmidt, Am. J. Physiol., 1924, 7I, 178. 
2. Nach der modernen Theorie, der Filtrations-Rückresorptionstheorie, ist Krreatinin eine Nichtschwellensubstauz (,non-threshold body“ nach Cushny), ${ }^{5)}$ d. h. Kreatinin, das durch Filtration mit Wasser und anderen Krystalloiden zusammen aus dem Blut in den Kapselraum übergegangen ist, wird beim Passieren durch die Harnkanülchen nie rückresorbiert, weder durch aktive Rückresorption noch durch passi ve Rückdiffusion. Bei der innerhalb eines bestimmten Zeitraums aufgefangenen Menge des Glomerulusfiltrats (GF) und des Harns (U) kann also die absolute Menge des Kreatinins sowohl im Harn als auch im Glomerulusfiltrat ganz unverändert bleiben. Also ist die absolute Menge des Kreatinins im Harn $(\mathrm{KrU} \times \mathbf{U})$ gleich der im Glomerulusfiltrat $(\mathrm{KrG} \times \mathrm{GF})$, aber $\mathrm{KrG}=\mathbf{K r P}$,

$$
\therefore \mathrm{KrP} \times \mathrm{GF}=\mathrm{KrU} \times \mathrm{U} \text { und } \mathrm{GF}=\frac{\mathrm{KrU} \times \mathrm{U}}{\mathrm{KrP}} \text {. }
$$

Vor Anwendung der Rehbergschen Methode wäre eigentlich das ihr zugrunde liegende Prinzip eingehend zu erörtern und verchiedene Einwïnde zu erledigen, aber wir brauchen nicht näher darauf einzugehen, da Rehbergis selbst mit Vorbedacht viele Einwïnde und Möglichkeiten erwogen und sie gründlich erörtert hat. Schon beim blossen Lesen seiner Beschreibung gewinnt man den Eindruck, dass seine Methode auf experimenteller und strengster theoretischer Basis aufgebaut ist, und nach den modernen Anschauungen uber Harnbildung scheint diese Messungsmethode für klinische $Z$ wecke sehr passend zu sein. Wir benutzten diese Methode mit einer kleinen Modifikation und haben die Menge des Glomerulusfiltrats zuerst bei Normalen und dann bei verschiedenen Kranken bestimmt. Hier seien nun die Beobachtungen an Normalen und Beriberikranken mitgeteilt.

Ausfubrung und Berechnung: Die Probe wurde stets morgens fruh im nüchternen Zustande ausgefuihrt und, um die Bedingungen des Versucbes konstant zu erbalten, erIaubten wir den Versuchspersonen keine Flussigkeitsaufnahme jedesmal von der Zeit nach dem Abendessen des rorangehenden Tages bis zur Versuchszeit am Morgen. Die Versuchsperson musste morgens früh Harn lassen und bekam sogleich $1 \mathrm{~g}$ Kreatinin, in $100 \mathrm{~cm}$ Wasser gelöst. Genau nach 60 Minuten wurde wieder Harn gelassen und gesammelt und sofort durch Venenpunktion eine Blutprobe entnommen, wobei wir als Antikoagulant Kaliumoxalat benutzten. Beim ersten und zweiten Irarnlassen musste die Versuchsperson ihre Blase rollständig entleeren, der zweite Harn war zur Bestimmung nötig. Dann wurde die Menge des innerhalb einer Stunde ausgeschiedenen Harns mittelst eines Messzylinders und die Konzentration des Kreatinins im betreffenden Harn und im Blutplasma nach Folin $\left.{ }^{6}\right)$ kolorimetri.ch bestimmt.

Die K reatininbestimmung wurde folgendermassen ausgeführt : Man tut 1,0 ccm Harn (einige $\mathrm{cm}$ bei verdiuntem Harn, soduss er ungefïhr 0,3-1,0 $\mathrm{mg}$ Kreatinin enthält) in einen $100 \mathrm{ccm}$ fassenden Messkolben und 1,0 ccm der Harnstandardlösung $(0,1 \mathrm{~g}$ chemisch reines Kreatinin in $100 \mathrm{~cm}{ }_{1}^{1} \mathrm{~N}$ Salzsüurelösung) in einen anderen. Jeder Kolben wird mit 20 ccm gesättigter wässeriger Pikrinsäure:ösung und 1,5 ccm 10\%iger Natronlauge beschickt und nach 10 Minuten mit destilliertem Wasser bis zur Marke gefillt, und diese beiden Lösungen werden sogleich kolorimetrisch rerglichen.

5) Cushny, vgl. 2) S. 28.

6) Folin, J. Biol. Chem., 1914, 17, 469. 
$1,0 \mathrm{ccm}$ Plasma und 1,0 cem Blutstandardlösung ( $1 \mathrm{mg}$ Kreatinin in $100 \mathrm{cem} \frac{1}{10} \mathrm{~N} \mathrm{Salz}-$ säurelösung) werden je in ein Reagenzgläschen getan, und jedes Reagenzgläschen wird mit 5 ccm gesïttigter wässeriger Pikrinsüurelösung versetzt und stark geschüttelt (Enteiweissung). Dana lässt man das Gernenge 20 bis 30 Minuten lang stehen, zentrifugiert und filtriert es darauf durch trocknes Filtrierpapier. Je $4 \mathrm{ccm}$ dieses Filtrats werden in ein anderes Reagenzglïschen getan, jedem $0,2 \mathrm{ccm} 10 \%$ iger Natronlaugelösung hinzugefüt und dies nach 10 Minuten kolorimetrich verglichen.

$\mathrm{U}_{\mathrm{m}}$ das Glomerulusfiltrat (GF) zu berechnen, benutzt man folgende Formel :

$$
\begin{aligned}
\mathrm{GF}=\mathrm{U} / 60 \times \mathrm{KrU} / \mathrm{KrP} \text {, wo } \\
\\
\mathrm{GF}=\mathrm{Glomerulusfiltrat} \text { pro Min. (cm), } \\
\mathrm{U}=\mathrm{H} \text { arnmenge pro Min. (cm), } \\
\mathrm{KrU}=\mathrm{mg} / \mathrm{dl} \text { des Kreatinins im Harn und } \\
\mathrm{KrP}=\mathrm{mg} / \mathrm{dl} \text { des } \mathrm{K} \text { reatinins im Plasma sind. }
\end{aligned}
$$

Nach den Versuchen ron Hoogenhuyse, Neubauer, ${ }^{8}$ ) Orlovius $s^{9}$ und nach der kürzlich veröffentlichten Abhandlung von $S$ ahe $k^{109}$ aus der chirurgischen Klinik der hiesigen Universitat ist es sehr wahrscheinlich, dass die Menge des innerhalb eines bestimmten Zeitraums ausgeschiedenen Kreatinins als Gradmesser der Nierenfunktion gelten kann. Wir haben also nebenbei die Menge des innerhalb der Versuchsstunde im Harn ausgeschiedenen Kreatinins berechnet.

\section{Glomerulusfiltrat bei Gesunden.}

Bei diesem Versuche dienten als Versuchspersonen 8 Männer von 18 bis 52 Jahren (Kollegen, Laboranten, Diener) und 8 Frauen von 17 bis 45 Jahren (Pflegerinnen, Dienerinnen), welche ganz gesund waren und anamnestisch keine Beriberi hatten.

In Tab. I sind die 16 Normalbestimmungen angeführt, in denen die durchschnittliche Menge Glomerulusfiltrat $177,1 \mathrm{~cm}$ pro Min. beträgt und die ausgeschiedene Menge des Kreatinins 0,2071 g. Nach dem Geschlecht ist der Wert des Mannes etwas höher als der der Frau ; nämlich der erste beträgt durchschnittlich $190,9 \mathrm{ccm}$ pro Min. und 0,2187 $\mathrm{g}$ und der letzte $163,2 \mathrm{ccm}$ pro Min. und 0,1954 g.

Nach dem Versuche Re hbergs' liegt die Menge des Glomerulusfiltrats bei gesunden Dänen zwischen 100-150 ccm pro Min. Wenn man also unser Resultat mit dem Rehbergs vergleicht, so findet man die Filtrationsmenge des Japaners etwas höher als die des Europüers, was vielleicht auf dem Unterschied der Nahrung und der Gewohnheit beruhen dürfte. Auf jeden Fall müssen wir annehmen, dass die Menge des nach dieser Methode berechneten Glomerulusfiltrats bei uns zwischen 150 und $200 \mathrm{~cm}$ pro Min. liegt.

$\mathrm{Ob}$ in den Glomeruli der menschlichen Niere eine so kolossale Filtration erfolgen kann oder nicht, hat schon Rehbergi) in seinen Abhand-

7) Hoogenhu y se, Ztrlbl. f. Chir., 1914, 41, 377.

8) Neubauer, Münch. med. Wochschr., 1914, 857.

9) Orlovius, Ztrlbl. f. Gebh. u. Gyn., 1915, 77, 348.

10) Saheki, Tohoku J. Exp. Med., 1929, 14, 146. 
Tabelle I.

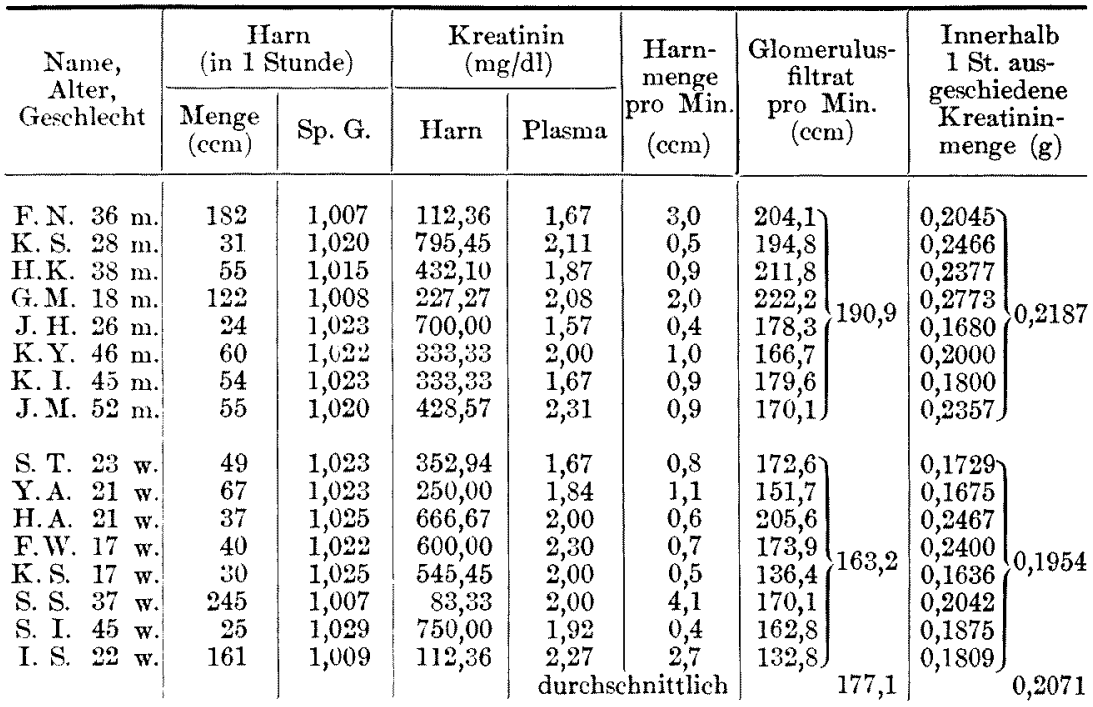

lungen vielfach erörtert und theoretisch bewiesen, dass diese Zahl keineswegs so unglaublich gross ist. Obwohl er nach den Versuchen von Vimtrup, ${ }^{11)}$ Hill, ${ }^{12)}$ Starling, ${ }^{3)}$ Haymann und Starr, ${ }^{13)}$ Landis ${ }^{14)}$ u.a. rein theoretisch und auf Grund seiner eigenen Versuche experimentell darauf hingewiesen hat, dass die Filtration von $150 \mathrm{ccm}$ pro Min. nicht unglaublich ist, können wir leider nicht sogleich die nach seiner Methode gewonnene $\mathrm{Zahl}$ als sichere Filtrationsmenge annehmen, da diese Methode teils auf experimenteller, teils aber auf theoretisch-by pothetischer Basis aufgebaut ist. Ja, solange man bei der Erklärung der Harnbildung nicht über Hypothesen hinauskommt, hat diese Methode einen schweren Nachteil. Wenn man andererseits daran denkt, dass die moderne FiltrationsRückresorptionstheorie im allgemeinen als höchstwahrscheinlich richtig angesehen wird, und dass, wenn diese Theorie unannehmbar wäre, die andere noch viel unglaublicher sein müsste, dann haben wir vielleicht recht, anzunehmen, dass die von uns berechnete Menge des Glomerulusfiltrats keineswegs ganz unwahrscheinlich ist. Von diesem Gedanken ausgehend sind

11) Vimtrup, Physiol. Paper ded. to Prof. Krogh, 1926 Kopenhagen, 357; Am. J. Anat, 1928, 41, 123.

12) Hill, J. Physiol, 1921, 54, Proc. Physiol. Soc., cxlit.

13) H a y man und St ar r, J. Exp. Med., 1925, 42, 641.

14) Land is, Am. J. Physiol., 1927, 81, 124; 82, 217. 
wir schliesslich zu der Ansicht gekommen, dass die von uns berechnete Zahl, weun sie auch nicht die wahre Zahl zeigt, doch dem wirklichen Filtrat sehr nahe steht. Jedenfalls glauben wir, dass diese Bestimmung relativ sehr interessante Ergebnisse zeitigen kann.

\section{Glomerulusfiltrat bei Beriberi.}

Es ist eine sichere Tatsache, dass die Menge des Harns in frischen oder akuten Fällen von Beriberi weistens deutlich abnimut und dass erhebliche Verminderung der Harnmenge ein Zeichen der schweren perniziösen Form ist. Wir beobachteten stets, dass sich die Harnmenge mit dem Eintritt der Besserung wieder hebt und die 24 stïndige Harnmenge über $2000 \mathrm{ccm}$, je sogar bis $4000 \mathrm{ccm}$ steigt und dass diese, wenn sich die Krankheit erneut verschlimmert, wieder sinkt.

Es gibt zahlreiche Untersuchungen und Theorien über die Störung des $W_{\text {asserhaushalts }}$ bei Beriberi, und es wird als sicher angenommen, dass die Hauptursache dieser Störung extrarenal zu suchen ist. Indessen kann man selbstrerständlich den renalen Einfluss nicht ganz ausschliessen, $\mathrm{d}$ a viele anatomisch-histologische und funktionelle Untersuchungen darüber ( Yamagiwa, ${ }^{15}$ ) Nagayo, ${ }^{16}$ ) Sassa, ${ }^{17}$ ) Kobayashi, ${ }^{18}$ ) Shimazono, ${ }^{19}$ ) T. Kato und seine $\mathrm{Schüle{ } ^ { 2 0 } )}$ ) vorliegen und verschiedene Theorien vorgebracht worden sind; aber es würde hier zu weit führen, auf Einzelheiten aus der betreffenden Literatur einzugehen.

Was nun die Frage anbelangt, wie sich die Harnmenge bei Beriberi vermindert, so sind hierbei natürlich zahlreiche Möglichkeiten in Erwägung zu ziehen, was schon $\mathrm{Yamaguchi}$, A be u. a. aus hiesiger Klinik ${ }^{20)}$ vielfach erörtert haben. Aber wenn man die Frage nur auf den Mechanismus der Ab- und Zunahme der Harnbildung in der Niere beschränkt, so kommen nach der Filtrations-Rückresorptionstheorie nur zwei Möglichkeiten in Betracht, d. h. die verminderte Glomerulusfiltration und die gesteigerte Rückresorption. Nun ist es natürlich eine sehr interessante und noch vollkommen offene Frage, ob sich die Menge des Glomerulusfiltrats bei Beriberi ebenso vermindert oder nicht.

19 Beriberikranke sind von uns beobachtet worden; es handelt sich um 5 rudimentäre, 5 leichte, sensibel-motorische, 6 mittelschwere und frische und 3 akute kardiale Formen. Tab. II gibt die Messungen des Glomerulusfiltrats bei den verschiedenen Formen der Beriberi wieder.

15) Yamagiwa, Tokio-Igakkai-Zasshi, 1892, 3, 427; 1900, 12, 635. (jap.)

16) Nag a yo, Verh. d. Jap. Path. Ges., 1912, 2, 19.

17) Sassa, Nihon-Naikagakkai-Zasshi, 1924, 12, 72. (jap.)

18) Ko y abash i, Tokio-Igakkai-Zasshi, 1924, 38, 295. (jap.)

19) Shimazono, Nisshin-Igaku, 1921, 11, 1. (jap.)

20) Yamaguchi, Tohoku J. Exp. Med., 1928, 10, 435; Hayasaka, Ibid., 1929, 14, 72; Kimura, Nibon-Naikagakkai-Zasshi, 1926, 16, 365 (jap.); A be, wird demnächst erscheinen. 


\begin{tabular}{|c|c|c|c|}
\hline  & 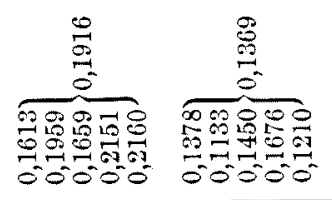 & 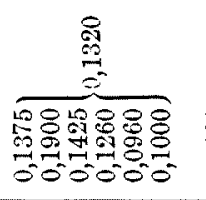 & 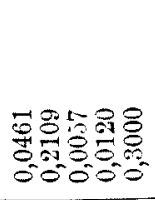 \\
\hline 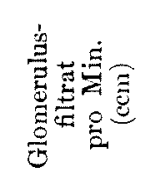 & 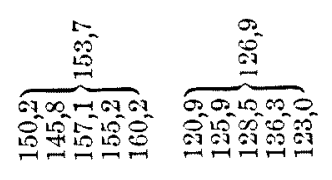 & 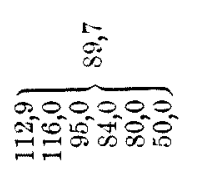 & 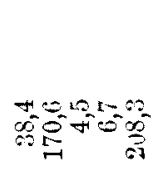 \\
\hline 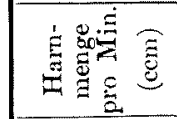 &  & $0+901=01=$ & $\Leftrightarrow=B=0$ \\
\hline \multirow{2}{*}{ 量焉 } & 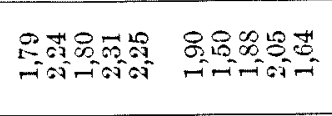 & 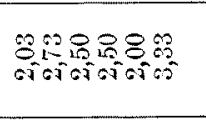 &  \\
\hline & 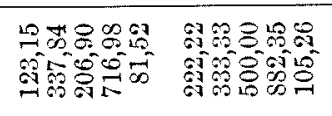 & 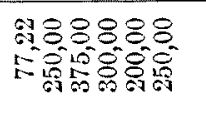 & 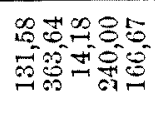 \\
\hline$\Xi$ & 900\% & 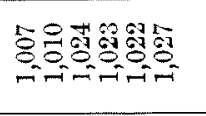 & 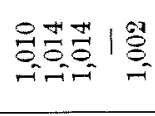 \\
\hline 吾 & 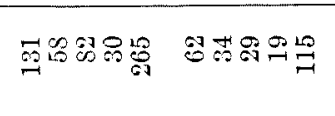 & 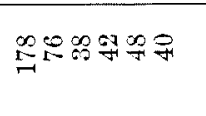 & 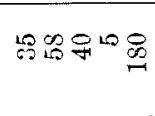 \\
\hline 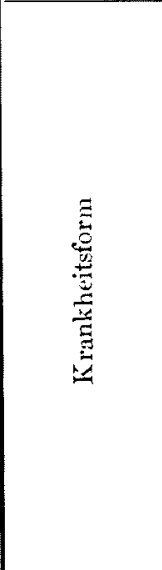 & 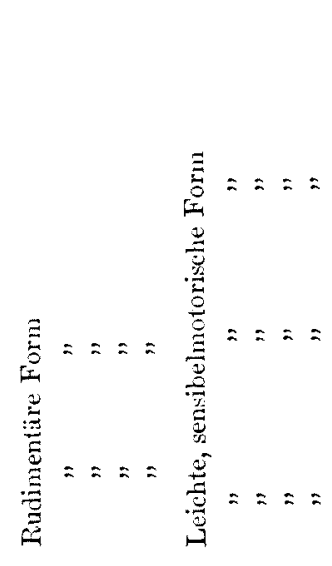 & 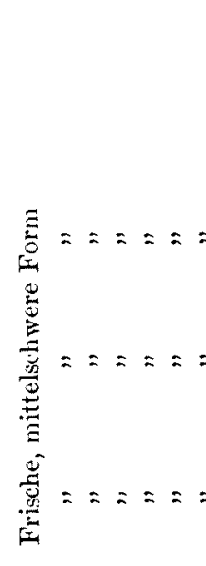 & 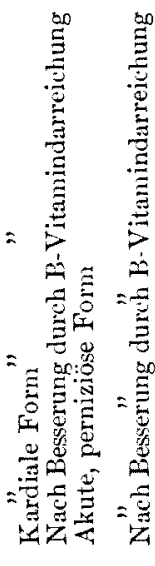 \\
\hline 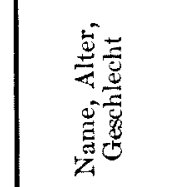 & 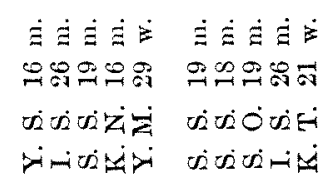 & 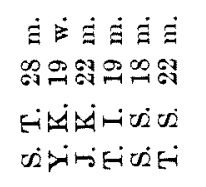 & 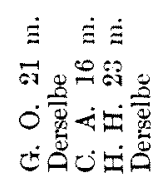 \\
\hline
\end{tabular}


Man sieht aus den Zahlen in der Tabelle, dass sich die Filtrationsmenge bei der rudimentären Form ein wenig (durchschnittlich 153,7 ccm pro Min.), bei der leichten, sensibel-motorischen, deutlicher (durchschn. $126,9 \mathrm{ccm}$ pro Min.), bei der mittelschweren ziemlich auffallend (durchschn. 89,7 cem pro Min.) und zuletzt bei akuten Fällen erheblich vermindert $(38,4,6,7$ und 4,5 ccm pro Min.). Nebenbei bemerkt, ist die Menge des ausgeschiedenen Kreatinins der Filtrationsmenge entsprechend vermindert. Es war ganz auffallend, dass die stark verminderte Filtratmenge und Kreatininausscheidung mit dem Eintritt der Besserung durch B-Vitamindarreichung sprungweise über die Norm hinaus zunahmen ; einige Beispiele davon sind in dem unteren Teil der Tab. II angegeben.

Aus diesen Tatsachen lässt sich nun folgern, dass die Verminderung der Harnmenge bei Beriberi hauptsächlich auf der Abnahme der Glomerulusfiltration beruhen muss, wobei diese als Gradmesser der Schwere der Krankheit gelten kann. Die Erklärung dafür, wie bei Beriberi, insbesondere bei frischen und kardialen Fällen eine so erhebliche Verminderung der glomerulären Filtration eintritt, ist sehr schwierig, aber so viel wenigstens kann man sagen, dass diese Verminderung in hohem Masse, durch extrarenale Störungen bedingt ist, weil das Glomerulusfiltrat eigentlich mit extrarenalen Faktoren, Blutdruck, kolloid-osmotischem Druck, chemischphysikalischer Beschaffenheit des Bluts und Permeabilität der Nierenglomeruli in inniger Beziehung steht. Die kardiale Insuffizienz wird natürlich eine Rolle dabei spielen, da die Filtration von der Blutströmung sehr abhängig ist, aber dass diese Abnahme der Filtration hauptsüchlich auf kardialer Störung beruht, glauben wir nicht, weil wir bei leichten und rudimentären Fällen gar keine Symptome kardialer Insuffizienz festgestellt haben, worüber wir später in der II. Mitteilung* dieser Studien, Glomerulusfiltrat bei Kreislaufinsuffizienz, nochmals berichten werden.

\section{Zusammenfassung.}

1. Die Menge des nach der oben beschriebenen Kreatininbelastungsmethode berechneten Glomerulusfiltrats gesunder Menschen beträgt in Durchschnitt $177,1 \mathrm{~cm}$ pro Min., wobei aber Männer einen etwas höheren $(190,9 \mathrm{~cm})$ und Frauen einen etwas niedrigeren Wert $(163,2 \mathrm{ccm})$ zeigen ; der Einfachheit halber kann man die Menge zwischen 150 und $200 \mathrm{~cm}$ pro Min. als normal annehmen.

*) Wird demnächst in dieser Zeitschrift erscheinen. 
2. Bei Beriberikranken findet man stets viel niedrigere Werte, insbesondere bei frischen oder kardialen Fällen sehr auffallend viel tiefere. Sie können oft unter $10 \mathrm{ccm}$ pro Min. betragen. Diese Verminderung der Glomerulusfiltration geht der Schwere der Krankheit parallel.

3. Die stark reduzierte Filtration kann mit dem Eintritt der Besserung durch B-Vitamindarreichung sprungweise zur Norm zurückkehren.

4. Die Menge des innerhalb einer Stunde ausgeschiedenen Kreatinins geht beinahe der Filtrationsmenge parallel.

Zum Schluss sei noch bemerkt, dass diese Bestimmung des Glomerulusfiltrats bei Beriberi diagnostisch und prognostisch von grosser Bedeutung ist. 